\title{
A number of blood biochemical parameters and endothelium- associated urine proteins of healthy people at head down bed rest
}

\author{
L.Kh. Pastushkova ${ }^{1 *}$, D.N. Kashirina ${ }^{1}$, A.G. Brzhozovsky ${ }^{1}$, A.M. Nosovsky ${ }^{1}$, \\ A.S. Kononikhin ${ }^{1,2}$, V.A. Ivanisenko ${ }^{3}$, E.S. Tiys ${ }^{3}$, E.N. Nikolaev ${ }^{2}$, I.M. Larina ${ }^{1}$ \\ ${ }^{1}$ Institute for Biomedical Problems - Russian Federation State Scientic Research Center, RAS, \\ Moscow, Russia \\ ${ }^{2}$ Institute of Energetic Problems of Chemical Physics RAS, Moscow, Russia \\ ${ }^{3}$ Institute of Cytology and Genetics SB RAS, Novosibirsk, Russia \\ *e-mail:lpastushkova@mail.ru
}

Key words: urine, chromatography-mass spectrometry, head-down bed rest, cardiovascular system, endothelial dysfunction

Motivation and Aim: Head down bed rest (HDBR), a strict bed rest with negative tilt of the head-end of the bed, triggers adaptive mechanisms of cardiovascular, endocrine, central and peripheral nervous systems. With regard to cardiovascular system, HDBR induces hypovolemia and cardiovascular deconditioning with alteration in vascular functions of various body regions, that is accompanied by endothelial dysfunction. Carbohydrate metabolism is also undergoing changes: insulin secretion increases and glucose tolerance is impaired. It is known that endothelial dysfunction is the first step in the development of atherosclerosis associated with insulin resistance syndrome. Therefore, we aimed to reveal the relationships between endothelium-associated proteins and the biochemical variables related to carbohydrate metabolism and its regulation.

Results: Mass spectrometry-based proteomics was employed to analyze urine samples from 8 healthy volunteers who remained at the bed rest study for 21 days, with an angle of inclination relative to the longitudinal axis of the body horizontal position $-6^{\circ}$. ANDSystem software which builds associative networks was used to identify urinary proteins functionally related to the endothelium. We identified 7 endothelium-related biological processes, directly linked to 13 urine proteins. Analysis of correlations with biochemical variables revealed a positive correlation between fasting blood glucose and the next urine protens: albumin, CD44 antigen, endothelial protein C receptor, mucin-1, osteopontin, receptor tyrosine kinase. As well, we found a positive correlation between HOMA-insulin resistance index and urine protens: endothelial protein $\mathrm{C}$ receptor and syndecan-4.

Conclusion: These results might suggest the involvement of above-mentioned proteins in glucose metabolism and their effects on carbohydrate metabolism. Thus, proteomics methods allowed obtaining new data about changes induced by HDBR, and suggesting the possible mechanisms of these changes.

Acknowledgements: The work was supported by RFBR grant No. 18-34-00524. We express special thanks to Bruker Daltonics for financial and technical support. 